\title{
Sexologia e adolescência
}

\author{
Gisela D. B. Balbino* \\ Ricardo G. Brasil* \\ Raphael C. Furtado* \\ Fabiana C. Telles* \\ Karen P. Pockel* \\ Vitor G. Flosi* \\ Sérgio A. Almeida*
}

\section{RESUMO}

Objetivos: Este trabalho é uma tentativa de realizar um levantamento analítico sobre as principais dúvidas de adolescentes do $2^{\circ}$ grau, em relação a sexo, excluída a relação deste com a AIDS especificamente.

Materiais e Métodos: Foram distribuídas, coletadas e analisadas 183 fichas de alunos de $1^{\circ}$ e $2^{\circ}$ colegial de uma escola da rede pública estadual, nas quais estes se identificavam apenas pela série, idade e sexo.

Além disso, foi relatado nas fichas, para um estudo empírico analítico, as duas principais dúvidas dos alunos em relação a sexo.

Resultados: Dos alunos que participaram do estudo, $61,2 \%$ eram do sexo feminino e $33,6 \%$ do sexo masculino. A idade mais freqüente foi de 15 anos, tanto no sexo feminino quanto no masculino $(35,7 \% ; 47.8 \%$

\footnotetext{
* Faculdade de Medicina de são José do Rio Preto. Departamento de Medicina Integrada. Disciplina de sexologia/Psiquiatria. 
respectivamente). $16,4 \%$ das fichas não continham perguntas, e das perguntas das fichas restantes $63,77 \%$ enquadravam-se nos quatro topicos mais freqüentes (Métodos anticoncepcionais, Tipos de relação sexual, Gravidez e D.S.T.).

Discussão: Apesar do comportamento sexual dos adolescentes ser um assunto de considerável especulação, ainda é muito pouco estudado. Deve-se então pensar em novas alternativas para a educação sexual.

\section{SUMMARY}

Objectives: This work is an attempt to accomplish a survey about the main doubts from teenagers in the secondary school, in relation to sex, without the relationship between sex and AIDS, specifically.

Material and methods: 183 students forms (secondary school) were distributed, taken back and analysed in a public school. The only topics were: grade, age and sex (no identification).

They had to write their two main doubts in relation to sex for an empiric analytical study.

Results: Out of these students that took place in this study, 61,2\% were feminine and $33,6 \%$ were masculine. The most frequent age was 15 years old, both feminine and masculine $(35,7 \%$ o; $47,8 \%$ o respectively).

There weren't any questions in $16,4 \%$ of the forms. And about the $63.77 \%$ left were relate to these four main topics: Contraceptive methods, Types of intercourse, Pregnancy and Transmissable Sexual Diseases.

Discussion: Although the sexual behaviour of the teenagers is a matter of much speculation, it is still object of very little study. We should think about another alternatives for sexual education, then.

\section{INTRODUÇÃO}

A adolescência é freqüentemente encarada como época de turbulência, mas tem tanto a possibilidade de ser uma época de prazer e felicidade quanto de ser uma passagem agitada para a idade adulta. Essa natureza paradoxal da adolescência está muito ligada à sexualidade.

Espera-se que o adolescente desenvolva e aceite independência emocional simultaneamente a capacitações na interação de preceitos éticos, competência em função intelectual e um sentido de responsabilidade social e pessoal. Não fosse suficiente tudo isso, o adolescente também pre- 
cisa confrontar-se com uma variedade de questões relacionadas à sexualidade, sejam como lidar com sensações sexuais novas ou mais poderosas, participar de vários tipos de comportamentos sexuais, conhecer o amor, evitar a gravidez indesejada ou definir os papéis sexuais apropriados à idade.

É importante conceber que a idade cronológica não determina o comportamento sexual do adolescente, influências relevantes dos contextos de fundo sócio-econômico, cultural e religioso contribuem extremamente para a tomada de decisões de âmbito sexual. Essas decisões também refletem a preparação psicológica individual, os valores pessoais, o raciocínio moral, o medo das conseqüências negativas e o envolvimento em ligações românticas, por parte dos adolescentes.

A atividade sexual durante a adolescência pode assumir diferentes sentidos. Existe uma variedade de razões que levam os adolescentes a serem sexualmente ativos: por prazer físico, a busca de novas experiências. como indicação de maturidade sexual, para assemelhar-se e serem aceitos por um grupo, para desafiar os pais ou a sociedade, como recompensa ou punição, como fuga à solidão, como escape a outras pressões.

Nas últimas décadas têm aumentado a incidência de relacionamento sexual entre adolescentes e as amplas discrepâncias observadas entre a atividade sexual masculina e feminina, não vigoram mais tão claramente. Dentre os fatores que contribuíram para essa mudança no Comportamento sexual adolescente temos a revolução de costumes iniciadas na década de 60, o fato dos meios de comunicação de massa passarem a utilizar mensagens de fundo erótico como veículo publicitário, a tendência a ser cada vez mais elevada a idade para o casamento, estimulando o relacionamento sexual pré-conjugal e um amadurecimento sexual cada vez mais precoce, fato este universalmente observado.

O ser humano conseguiu evoluir para o ato sexual sem a necessidade de procriar, como conseqüência tem-se a divisão entre sexo, para reprodução e sexo para a recreação ou prazer. Porém, a sociedade ainda não se adaptou a esta revolução dos valores morais em relação à sexualidade; e isto vem fazendo com que pais e figuras em posição de autoridade (como a igreja) exerçam pressão nos jovens, no sentido de forçar o sexo para fora da área de recreação/prazer. Nesse contexto, o adolescente fica relegado a segundo plano, sem acesso a informações, educação sexual e ao esclarecimento de suas dúvidas e anseios. 


\section{OBJETIVOS}

Este trabalho é uma tentativa de realizar um levantamento analítico sobre as principais dúvidas de adolescentes do $2^{\circ}$ grau, em relação a sexo, excluída a relação deste com a AIDS especificamente.

\section{MATERIAIS E MÉTODOS}

Foi realizado um sorteio aleatório entre as duas Delegacias de Ensino de São José do Rio Preto. A partir disso, foi realizado um novo sorteio entre as escolas que a delegacia sorteada abrange. Com este critério, foram distribuídas fichas a 183 alunos de $1^{\circ}$ e $2^{\circ}$ colegial na EEPSG Prof. José Felicio Miziara.

Como identificação, os alunos preencheram estas fichas com suas respectivas série, idade e sexo. Além disso, foi pedido aos alunos que relatassem quais eram duas de suas principais dúvidas em relação a sexo. Foram consideradas apenas as duas primeiras perguntas por ficha, sendo desprezadas as demais.

Após a coleta desses dados, foi feito um estudo empírico analítico, onde as perguntas foram agrupadas de acordo com seus tópicos principais, sendo selecionados para posterior análise, os quatro tópicos mais freqüentes (Métodos Anticoncepcionais. Tipos de Relação Sexual, Gravidez e D.S.T.), e desprezados os demais tópicos (Masturbação, Impotência, Aborto, Orgasmo, Homossexualismo, Sexo/desejo/amor, Anatomia, Fisiologia, Tabus, Abuso sexual, Corrimento, e as perguntas que não se enquadravam nesses tópicos relacionados).

\section{RESULTADOS}

Dos 183 alunos que preencheram as fichas, $61,2 \%$ eram do sexo feminino e $36,6 \%$ do sexo masculino. Distribuem-se na faixa etária de 14 a 19 anos, de acordo com a seguintes porcentagens: 14 anos (14,2\%), 15 anos (39.3\%), 16 anos (30,1\%) 17 anos (9,8\%), 18 anos (2,2\%) e 19 anos (O.5\%). A idade mais freqüente em relação ao sexo feminino é a de 15 anos com $35,7 \%$, o mesmo acontece com o sexo masculino com $47,8 \%$. 
Das fichas coletadas 16,4\% não continham perguntas, $10,38 \%$ apenas uma pergunta e $73,22 \%$ duas ou mais perguntas, sendo que destas apenas as duas primeiras foram consideradas.

Das perguntas analisadas pelos autores, $63.77 \%$ correspondem àquelas enquadradas nos quatro tópicos mais freqüentes. Portanto, do total de perguntas analisadas, a porcentagem de aparecimento desses tópicos é a seguinte: Métodos anticoncepcionais 20,98\% (56 perguntas), Tipos de relação sexual $18,11 \%$ (48 perguntas). Gravidez $13,16 \%$ (35 perguntas) e D.S.T. $11,52 \%$ (31 perguntas).

As perguntas que foram enquadradas nesses tópicos estão expostas na tabela anexa. 


\section{TABELA DE PERGUNTAS DOS QUATRO PRINCIPAIS TÓPICOS}

\begin{tabular}{|c|c|c|c|}
\hline $\begin{array}{c}\text { Métodos } \\
\text { Anticoncepcionais } \\
(\mathbf{2 0 , 9 8 \%}) \\
\end{array}$ & $\begin{array}{c}\text { Tipos de } \\
\text { Relação Sexual } \\
(18,11 \%)\end{array}$ & $\begin{array}{l}\text { Gravidez } \\
(13,16 \%) \\
\end{array}$ & $\begin{array}{c}\text { D.S.T. } \\
(11,52 \%)\end{array}$ \\
\hline Quais são? & * Sexo Oral: & $/ 1^{\mathrm{a}}$ relação & Quais são? \\
\hline Qual o mais seguro? & O que é? & /menstruação & $\begin{array}{l}\text { Como se } \\
\text { manifestam? }\end{array}$ \\
\hline Como utilizar? & / virgindade & $\begin{array}{l}\text { /antes da } 1^{\mathrm{a}} \\
\text { menstruação }\end{array}$ & Como diagnosticar? \\
\hline \multirow[t]{15}{*}{ Conseqüencias do aso } & I doenças e riscos & /sexo oral & $\begin{array}{l}\text { Meios de } \\
\text { transmissão }\end{array}$ \\
\hline & * Sexo Anal: & /sexo anal & Meios de prevenção \\
\hline & O que é? & /idade & Consequiencias \\
\hline & / virgindade & /consanguinidade & \\
\hline & / doenças e riscos & /adolescência & \\
\hline & / prazer para a mulher & período fértil & \\
\hline & * Sexo Vaginal: & gravidez ectópica & \\
\hline & O que é? & Como diagnosticar? & \\
\hline & / virgindade & & \\
\hline & / menstruação & & \\
\hline & $/ \mathrm{Na}^{\mathrm{a}}$ relação: & & \\
\hline & O que acontece? & & \\
\hline & $\begin{array}{l}\text { /sangramento } \\
\text { /dor } \\
\text { /riscos }\end{array}$ & & \\
\hline & $\begin{array}{l}\text { /conseqüências } \\
\text { psicológicas }\end{array}$ & & \\
\hline & $\begin{array}{l}\text { /romprimento de } \\
\text { hímen (tipos e formas } \\
\text { de hímen) }\end{array}$ & & \\
\hline
\end{tabular}




\section{TABELA: DISTRIBUIÇÃO POR SEXO}

\begin{tabular}{c|c|c} 
SEXOF & SEXOM & Sem Informação \\
\hline $112(61.2 \%)$ & $67(36.6 \%)$ & $4(2,2 \%)$
\end{tabular}

TABELA: DISTRIBUIÇÃO POR IDADE

\begin{tabular}{c|c}
\hline IDADE & \\
\hline 14 anos & $26(14.2 \%)$ \\
\hline 15 anos & $72(39.3 \%)$ \\
\hline 16 anos & $55(30.1 \%)$ \\
\hline 17 anos & $18(9.8 \%)$ \\
\hline 18 anos & $4(2.2 \%)$ \\
\hline 19 anos & $\frac{1(0.5 \%)}{7(3.8 \%)}$ \\
\hline Sem Informação &
\end{tabular}

\section{DISTRIBUIÇÃO POR SEXO}

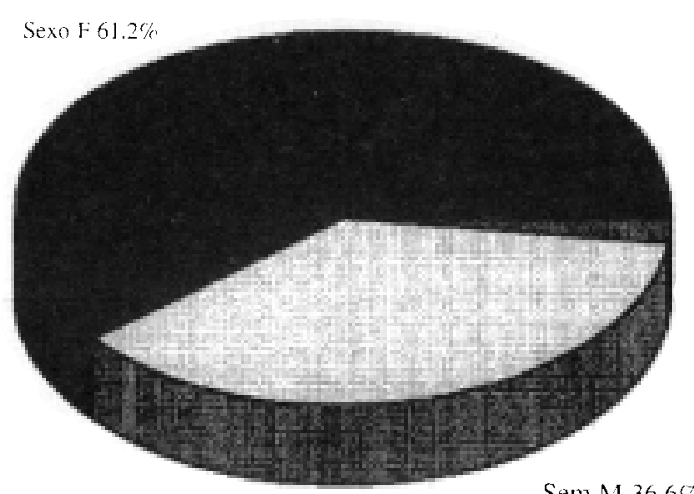

Sem Informação $2.2 \%$ 
TABELA: SEXO / IDADE

\begin{tabular}{c|c|c}
\hline IDADE & SEXOF & SEXO M \\
\hline 14 anos & $19(17,0 \%)$ & $7(10,4 \%)$ \\
\hline 15 anos & $40(35.7 \%)$ & $32(47,8 \%)$ \\
\hline 16 anos & $38(33.9 \%)$ & $17(25,4 \%)$ \\
\hline 17 anos & $11(9.8 \%)$ & $7(10,4 \%)$ \\
\hline 18 anos & $2(1.8 \%)$ & $2(3,0 \%)$ \\
\hline 19 anos & $1(0,9 \%)$ & 0 \\
\hline Sem Informação & $1(0,9 \%)$ & $2(3,0 \%)$
\end{tabular}

\section{DISTRIBUIÇÃO POR IDADE}

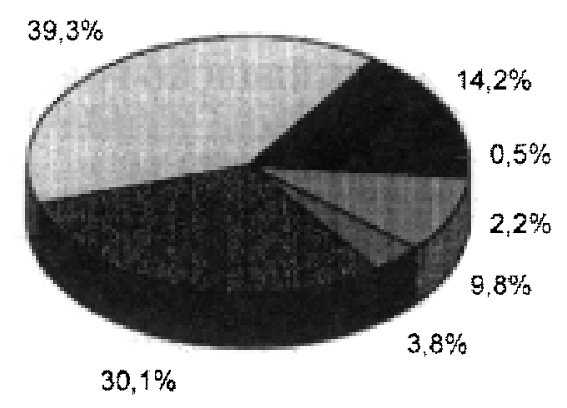

14 ANOS $\square 15$ ANOS 16 ANOS 17 ANOS 18 ANOS 19 ANOS 


\section{SEXO / IDADE}

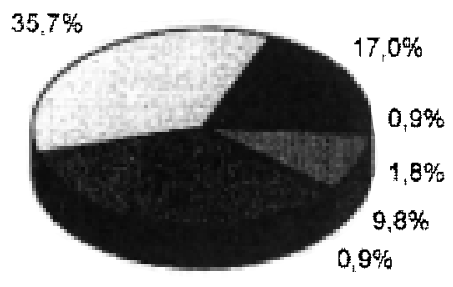

$33,9 \%$

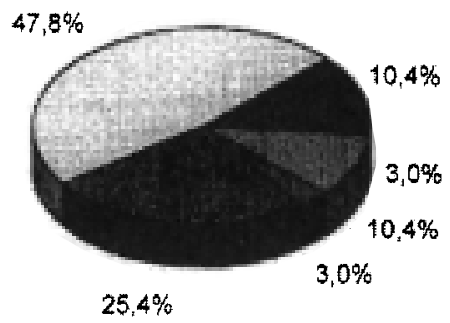

SEXOM

SEXOF

14 ANOS $\square 15$ ANOS 16 ANOS 畻 SEM INFORMAÇÃO [17 ANOS 18 ANOS 19 ANOS

\section{TABELA: DISTRIBUIÇÃO POR SEXO}

(ALUNOS DE $1^{\circ}$ COLEGIAL)

\begin{tabular}{c|c|c} 
SEXOF & SEXO M & Sem Informação \\
\hline $71(62,3 \%)$ & $40(35,1 \%)$ & $3(2,6 \%)$
\end{tabular}




\section{DISTRIBUIÇÃO POR SEXO \\ ( $1^{\circ}$ COLEGIAL)}

\section{Sexo F $62.3 \%$}

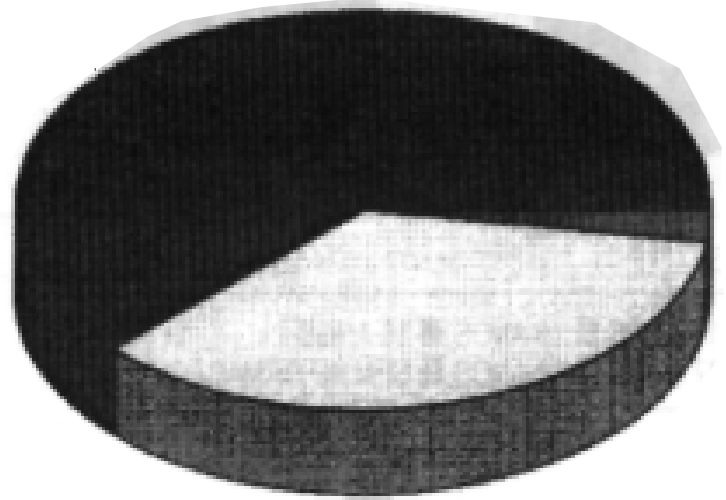

Sem Informação $2 \%$

Sem M 35, $1 \%$

\section{TABELA: SEXO / IDADE ( $1^{\circ}$ COLEGIAL)}

\begin{tabular}{c|c|c} 
IDADE & SEXO F & SEXO M \\
\hline 14 anos & $19(26,8 \%)$ & $7(17,5 \%)$ \\
\hline 15 anos & $28(39,4 \%)$ & $23(57,5 \%)$ \\
\hline 16 anos & $16(22,5 \%)$ & $5(12,5 \%)$ \\
\hline 17 anos & $7(9,9 \%)$ & $4(10,0 \%)$ \\
\hline 18 anos & 0 & $1(2,5 \%)$ \\
\hline Sem Informação & $1(1,4 \%)$ & 0
\end{tabular}




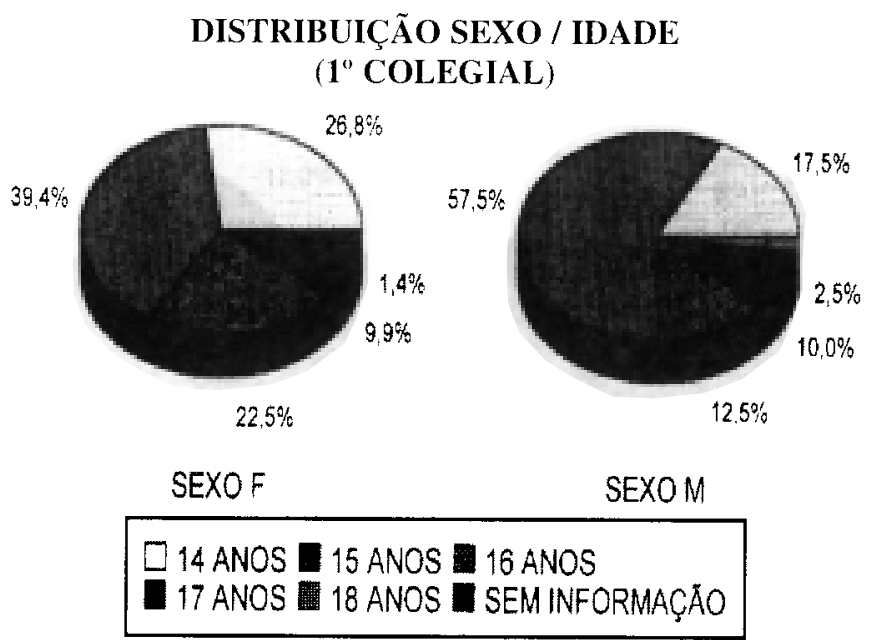

\section{TABELA: DISTRIBUIÇÃO POR SEXO (ALUNOS DE $2^{\circ}$ COLEGIAL)}

\begin{tabular}{c|c|c} 
SEXOF & SEXOM & Sem Informação \\
\hline $41(59.4 \%)$ & $27(39.1 \%)$ & $1(1.4 \%)$
\end{tabular}

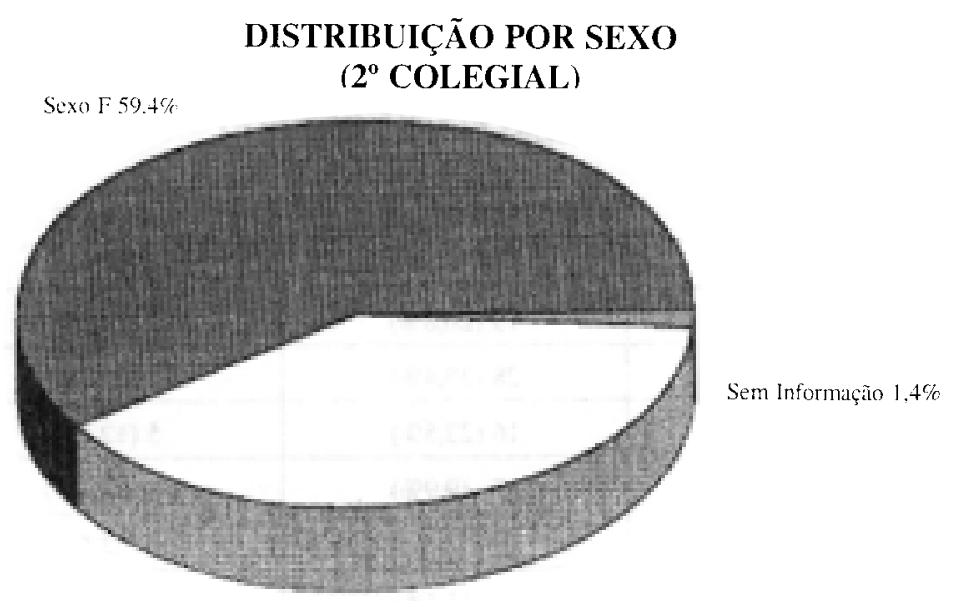

Sen $M 39.1 \%$ 


\section{TABELA: SEXO / IDADE ( $2^{\prime \prime}$ COLEGIAL)}

\begin{tabular}{c|c|c} 
IDADE & SEXO F & SEXO M \\
\hline $15 \operatorname{anos}$ & $12(29.3 \%)$ & $9(33.3 \%)$ \\
\hline $16 \operatorname{anos}$ & $22(53,7 \%)$ & $12(44.4 \%)$ \\
\hline $17 \operatorname{anos}$ & $4(9,8 \%)$ & $3(11.1 \%)$ \\
\hline $18 \operatorname{anos}$ & $2(4.9 \%)$ & $1(3,7 \%)$ \\
\hline $19 \operatorname{anos}$ & $1(2,4 \%)$ & 0 \\
\hline Sem Informação & 0 & $2(7.4 \%)$
\end{tabular}

\section{DISTRIBUIÇÃO SEXO / IDADE ( $2^{\circ}$ COLEGIAL)}
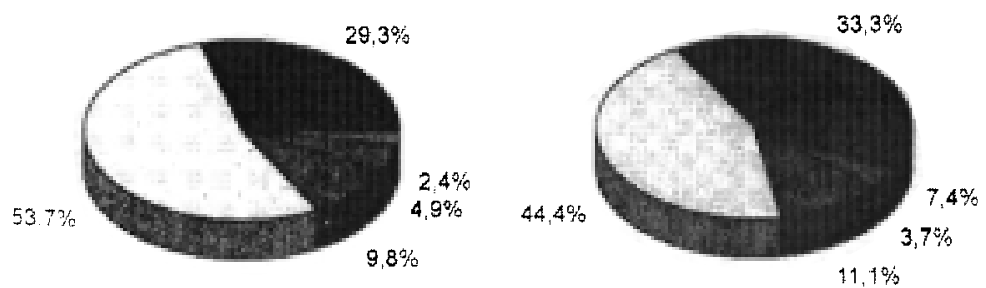

SEXOF

SEXOM

14 ANOS 15 ANOS 16 ANOS

17 ANOS 18 ANOS $\square$ SEM INFORMAÇĂO 


\section{DISCUSSÃO}

Após análise dos resultados, onde 83,6\% dos alunos revelaram dúvidas a respeito de sexo, concluímos que apesar do comportamento sexual dos adolescentes ser um assunto de considerável especulação, ainda é muito pouco estudado. Isso pode ser explicado, talvez, pela pouca atenção dada à educação sexual no país.

Uma educação cujo objetivo é desenvolver o auto-conhecimento, a preparação psicológica individual, os valores pessoais e o raciocínio moral, para a compreensão da sexualidade em seus aspectos também afetivos, culturais e estéticos; sem se descuidar da gravidez na adolescência e das D.S.T.s. Uma educação sexual que procura conduzir um diálogo com os adolescentes e dar-lhes instrumentos para que façam melhores opções em suas vidas e que os coloquem em harmonia consigo mesmos e com o ambiente em que estão inseridos.

\section{REFERÊNCIAS BIBLIOGRÁFICAS}

1. Adolescência, os Jovens e Nós: Uma Visão Pessoal - $2^{\mathrm{a}}$ parte: Um Ser Esquisito, Jornal de Pediatria (Rio de Janeiro); 61(2): 132, 137-8, 141-2, agosto 1986.

2. Comportamento Anticonceptivo na Adolescência, Revista Médica do Hospital São Vicente de Paulo; 5(12): 29-31, maio-agosto 1993.

3. Manual de Medicina Sexual; Kolodny, R. C., Masters, W. H., Johnson, V. E. Editora Manole, 1982.

4. O Relacionamento Amoroso; Kolodny, R. C., Masters, W. H., Johnson, V. E. Editora Nova Fronteira, 1988.

5. Sexualidade na Adolescência, Femina; 12(9): 825-35, 1984. 